\title{
Short-term wind speed forecasting system using deep learning for wind turbine applications
}

\author{
Gökhan Erdemir ${ }^{1}$, Aydın Tarık Zengin ${ }^{2}$, Tahir Cetin Akinci ${ }^{3}$ \\ ${ }^{1}$ Department of Electrical and Electronics Engineering, Istanbul Sabahattin Zaim University, Turkey \\ ${ }^{2}$ Department of Computer Engineering, Istanbul Sabahattin Zaim University, Turkey \\ ${ }^{3}$ Department of Electrical Engineering, Istanbul Technical University, Turkey
}

\begin{tabular}{l} 
Article Info \\
\hline Article history: \\
Received Mar 16, 2020 \\
Revised Apr 29, 2020 \\
Accepted May 27, 2020 \\
\hline
\end{tabular}

\section{Keywords:}

Deep learning

Short-term forecasting

Wind speed

Wind speed forecasting

Wind turbine

\begin{abstract}
It is very important to accurately detect wind direction and speed for wind energy that is one of the essential sustainable energy sources. Studies on the wind speed forecasting are generally carried out for long-term predictions. One of the main reasons for the long-term forecasts is the correct planning of the area where the wind turbine will be built due to the high investment costs and long-term returns. Besides that, short-term forecasting is another important point for the efficient use of wind turbines. In addition to estimating only average values, making instant and dynamic short-term forecasts are necessary to control wind turbines. In this study, short-term forecasting of the changes in wind speed between 1-20 minutes using deep learning was performed. Wind speed data was obtained instantaneously from the feedback of the emulated wind turbine's generator. These dynamically changing data was used as an input of the deep learning algorithm. Each new data from the generator was used as both test and training input in the proposed approach. In this way, the model accuracy and enhancement were provided simultaneously. The proposed approach was turned into a modular independent integrated system to work in various wind turbine applications. It was observed that the system can predict wind speed dynamically with around 3\% error in the applications in the test setup applications.
\end{abstract}

Copyright @ 2020 Institute of Advanced Engineering and Science. All rights reserved.

\section{Corresponding Author:}

Gökhan Erdemir,

Department of Electrical and Electronics Engineering,

Istanbul Sabahattin Zaim University,

Halkali, Kucukcekmece, Istanbul 34303, Turkey.

Email: gokhan.erdemir@izu.edu.tr

\section{INTRODUCTION}

Wind is one of the most promising ones among renewable energy sources [1]. The installed wind energy capacity of the world has been increasing exponentially in recent years. This increase causes wind energy to be an area of interest for investors and thus turns into a big business [1-5]. However, a lot of research is being done to decrease wind energy installation and energy production costs. These studies focus on improving the efficiency of power systems as well as improving the mechanical parts of wind turbines. Wind, which is the most popular energy source among renewable energy sources, has different difficulties compared to the region established in electricity generation. However, the most important problem of all wind farm operators is still wind speed, power estimation and turbine control [4-10].

Connection and operation of the generated energy to the interconnected grid have different problems. Initial investment costs of the power plants set up to convert wind speed into electrical energy are very high. This investment cost requires a detailed analysis of the wind speed data of the installation area. These analyses can be separated into two categories as short-term and long-term forecasting [11]. Short-term forecasts are up to several weeks, while long-term forecasts cover up to several decades. In the literature, 
many methods have been used to make short- and long-term forecasts from wind speed data. Most of the methods use statistical analysis techniques. Time series modeling techniques such as ARIMA or neural networks have been widely used in most studies for the prediction of wind speed [12-16].

Statistical learning method; estimates wind speed by deducting control system data and similar historical data [11]. The model uses for prediction is usually a time series model and estimates parameters using some iterations on the dataset. In some studies, data mining algorithms have also been used for prediction. Similar learning algorithms are used in data mining, just as they are used in artificial neural networks $[11,16,17]$. Statistical learning methods are preferred for two main reasons, which are prediction models that are easy to create and have high prediction accuracy for the short-term. Models based on physical systems can also be used in wind forecasting. These methods consist of a series of differential equations and are used for numerical weather forecasts [11]. These physical methods can be used in long-term systems that require more detailed information. Also, when these physical systems are compared with the estimations made using statistical learning methods, success rates are lower. However, there are approaches that use both the physical model and statistical models together. These are called hybrid model approach, where numerical weather forecast data is solved using statistical models. In general, multi-input like wind speed, direction, and other meteorological data are used for inputs of the prediction algorithm [18-21].

The Artificial Intelligence (AI) method offers very successful results thanks to its ability to map nonlinear features. The main advantage of AI methods is to estimate possible wind speed series without a predefined mathematical tool. As the representative of artificial intelligence methods, artificial neural networks [22, 23], feed-forward neural network [24] and adaptive linear element neural network for wind speed prediction are currently used by researchers [24-26].

Deep learning algorithms are preferred over traditional neural networks to reveal more detailed features of wind speed series. Deep learning algorithms provide very successful results in many computerbased sensing, vision, speech and signal processing [27]. A deep learning method has been applied to reveal the complex features of wind speed data [28-30]. Experimental results show that deep learning algorithms have the best performance among comparison models. While many analyses in wind speed prediction studies produced the predicted wind speed values directly from the raw wind speed data, the non-stationary effects of the wind speed series were also ignored. By comparison, deep learning is a subset of machine learning. However, although deep learning, and machine learning function similarly, their capabilities are different. deep-learning automatically finds the features to be used for classification, while machine learning provides these features manually. In addition, compared to machine learning, deep learning requires stronger processors and larger training data for its results. Compared to ANN, deep learning offers more layers working [31-37].

In this study, a short-term wind speed forecasting system is proposed using deep learning for wind turbines applications. In section 2, the system architecture and synthetic data generation are explained. Then the short-term wind speed forecasting system is described in the same section. Short-term wind speed forecasting results are presented in Section 3. Finally, conclusions are presented in section 4.

\section{THE PROPOSED SYSTEM ARCHITECTURE}

Global wind data at a 2.5-degree resolution have been published by the National Centres for Environmental Information, national oceanic and atmospheric administration (NOAA) [38]. Daily windvelocity-averages since 1948 have been given in the dataset provided by NOAA. In order to simulate a realistic situation, a data generation model was designed using the daily average wind-velocity values from the dataset and generated random values having the final average value was still consistent with the dataset. We hereby interpolated the daily average values to minute-by-minute values.

The data generation model was designed in Simulink and is shown in Figure 1. In this model, data generated by the method explained above were applied to a PID control system which controls a DC motor emulating the wind-turbine. A quadrature encoder was connected to the rotor measuring the rotational speed of the motor. Interpolated wind-data were simulated by this actual system and 4 hours of real-time data were collected for each day of each year. Therefore, we could be able to generate more detailed data that is consistent with the dataset. In the Simulink model given below, Encoder block measured the angular velocity of the rotor. Angular velocity than was fed to the PID Controller block as the feedback value. Rotor speed was generated using the actual data received from the data block and summed with a random value generated by the Random Number block. Time Pulses block generated timing pulses for each minute and hour in order to proceed to the next value in the dataset. Selector block used the hour pulse to pick the next value from the dataset. 


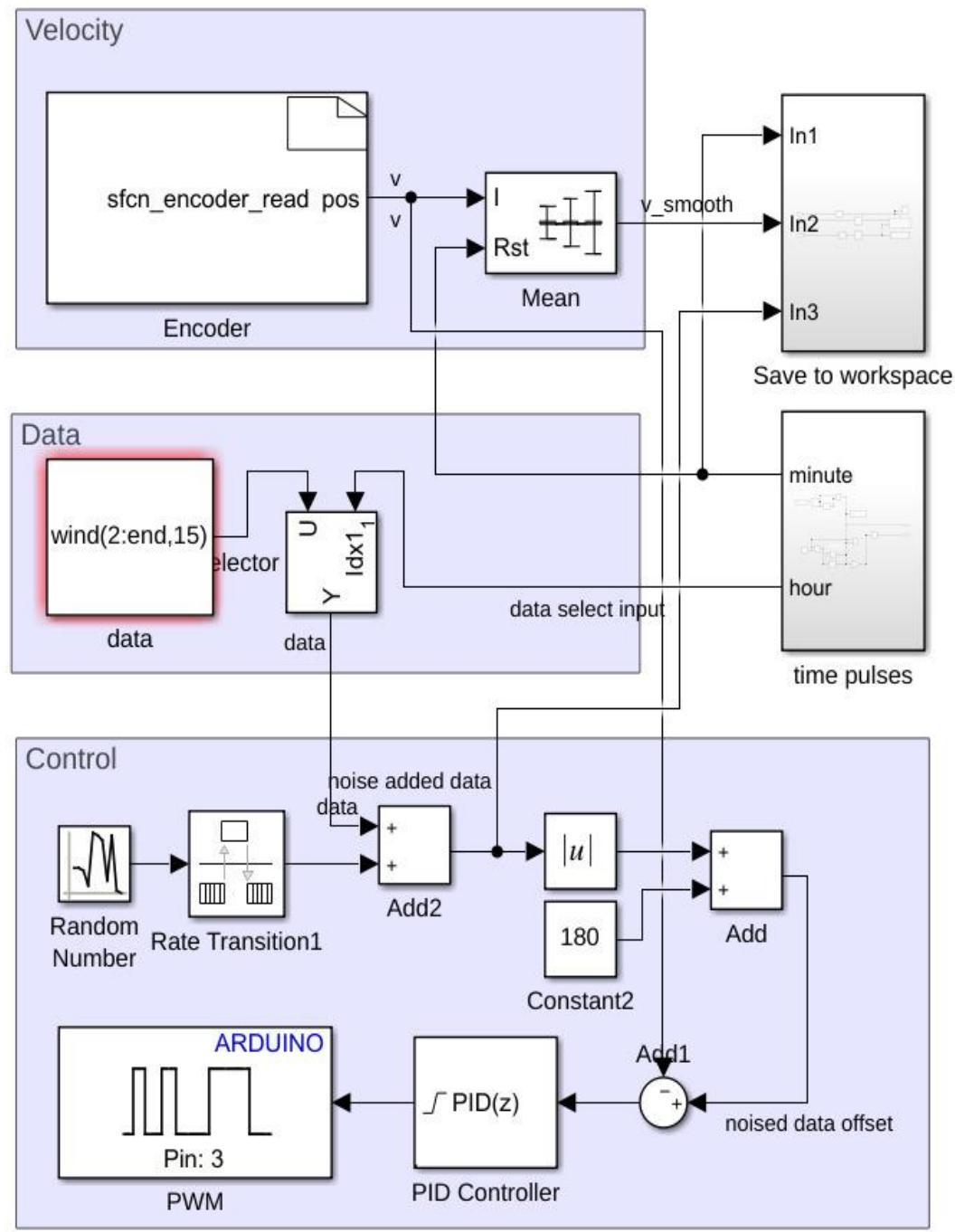

Figure 1. Interpolation/generation model

\section{THE SHORT-TERM WIND SPEED FORECASTING SYSTEM}

In this section, a short-term wind speed prediction was performed with two datasets, separately. KERAS library was used for the training model. 15 iterations were performed for network training with 15 minutes of data from both datasets. The input layer of the deep learning model consisted of a single neuron with linear activation. The hidden layer was built as the hidden LSTM layer by using 128 neurons with ReLU (Rectified Linear Unit) activation function for each neuron. The output layer was built as the hidden dense layer by using a single neuron with a linear activation function. Nvidia Jetson Nano microcomputer which directly connected to the motor output was used for both learning and forecasting. Thus, it will be easier to transfer the forecast results to any unit in the wind turbine and to process accordingly. Test results are presented in Section 4.

$$
e_{v}=\left|v_{a v g}+\alpha\right|_{i}-v_{a c t}
$$

Wind speed error $e_{v}$ is given in (1) where $v_{\text {avg }}$ was the daily average wind speed, $\alpha$ was a random number representing the daily fluctuation of wind speed, $v_{a c t}$ was the actual wind speed measured by the quadrature encoder, and $i$ was the index marking every minute. $e_{v}$ was fed to the PI controller running the DC motor emulating the turbine and synthetic data based on actual wind speed data were collected by the system minute by minute. Using the model given above two synthetic datasets were generated for every $15^{\text {th }}$ January with 1 minute and 6 minutes intervals, as shown in Figures 2 and 3, respectively. 


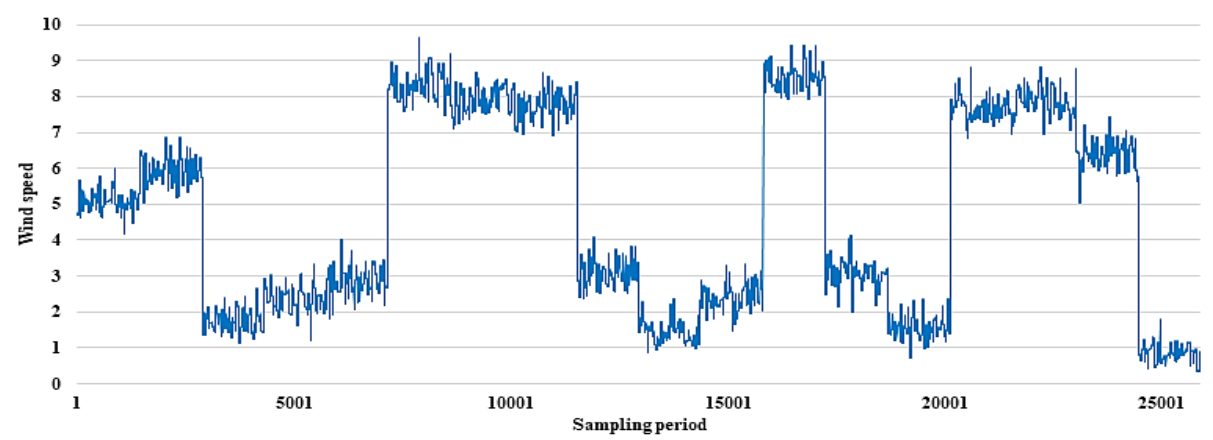

Figure 2. Generated data with a 1-minute time interval

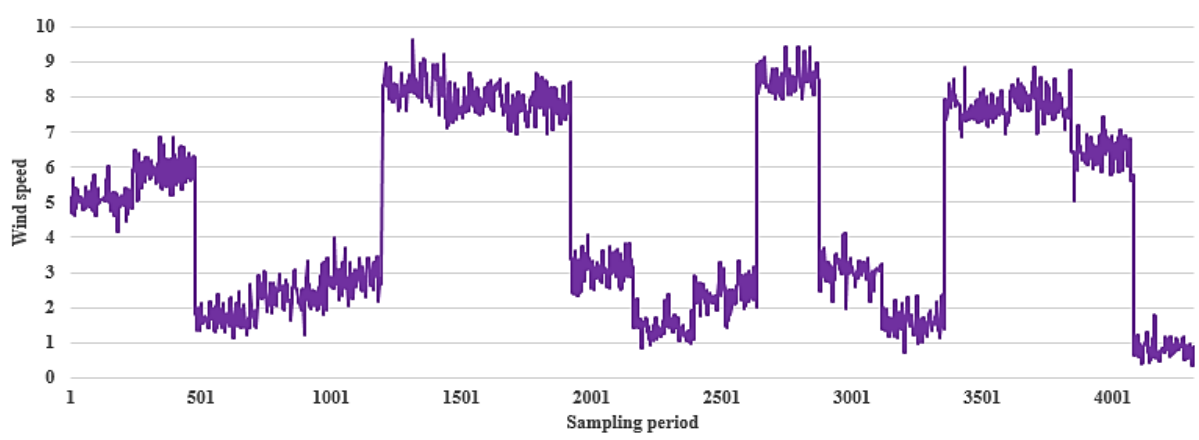

Figure 3. Generated data with 6 minutes time interval

\section{RESULTS AND DISCUSSION}

In this section, the test results of the system are presented. The test result after data training for the first dataset is shown in Figure 4. For a 1-minute-interval dataset, 30\% (approximately 5100 singular data) of the data were used for the test. According to test results, the proposed algorithm has a $3.315 \%$ prediction error rate. Using a 6-minute-interval dataset, again 30\% (approximately 850 singular data) of the data were used for the test, as well. The test results are shown in Figure 5. According to test results, the proposed algorithm has a $10.93 \%$ prediction error rate. According to the test results, the system was observed to produce better forecasting results with approximately $3.315 \%$ forecasting error by using the first dataset which has data with a 1-minute time interval. However, time and space complexity are higher than in the second dataset. The forecasting result had an acceptable error rate (approximately 10.93\%) using the second dataset. Therefore, the second set can be preferred for low time and space complexity due to the dynamic structure of the system. Computational costs were not a decisive factor because data would be gathered and calculated in a separate unit for both training and forecasting. The proposed approach had acceptable error rates with both datasets for wind turbine applications. In cases where data storage is limited, a 6-minute time interval dataset may be preferred. If there is no such limitation, it will be appropriate to use a 1-minute time interval data.

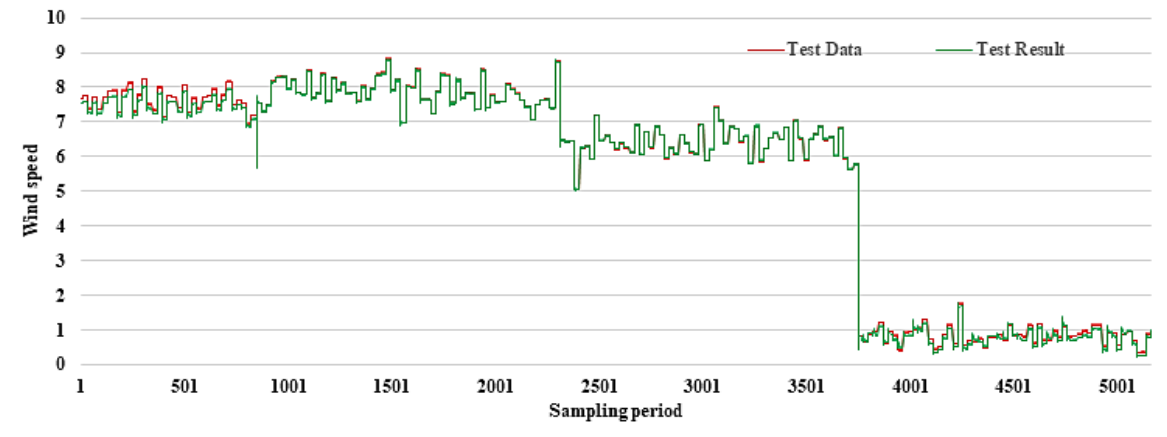

Figure 4. Test results with a 1-minute time interval 


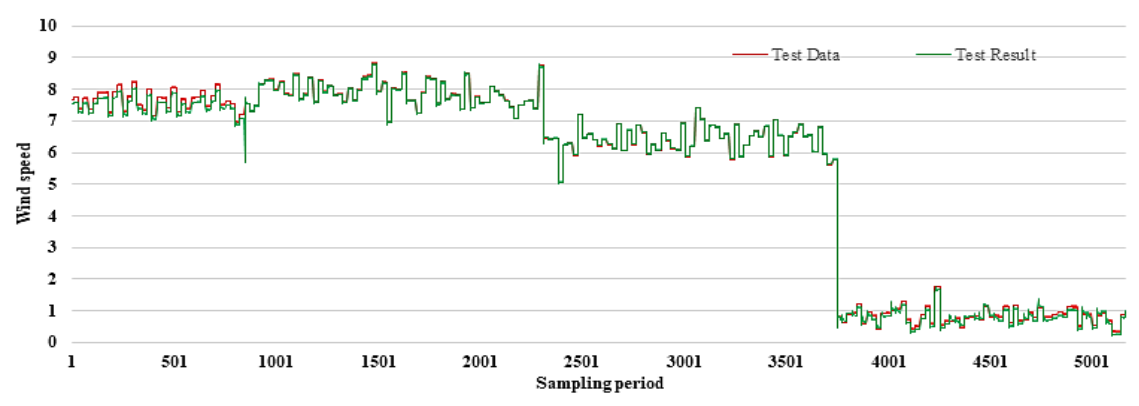

Figure 5. Test results with 6 minutes time interval

\section{CONCLUSION}

In this study, the short-term wind speed forecasting system using deep learning for wind turbines applications is presented. The proposed system produces short-term wind speed forecasts to be used in different kinds of wind turbine applications. The system can be easily integrated into wind turbines. Thus, it can be used as a brake system, emergency warning, etc. According to test results which were presented in section 3, it could be seen that the proposed approach produced better results using 1-minute time interval data for training. Moreover, the error rate achieved when using the 6-minute time interval data is also acceptable. The most important differences between the two datasets which were shown in Figures 4 and 5 , were error rates and space complexity. These criteria should be kept in mind when integrating the proposed system into actual applications.

\section{REFERENCES}

[1] N. L. Panwar, S. C. Kaushik, and S. Kothari, "Role of Renewable Energy Sources in Environmental Protection: A Review," Renewable and Sustainable Energy Reviews, vol. 15, pp. 1513-1524, 2011.

[2] S. H. Kulkarni, T. R. Anil, and R. D. Gowdar, "Wind Energy Development in India and a Methodology for Evaluating Performance of Wind Farm Clusters," Journal of Renewable Energy, vol. 2016, pp. 1-16, 2016.

[3] M. Junginger, et al., "Offshore wind energy (Chapter 7) in Technological Learning in the Transition to a LowCarbon Energy System,” Academic Press, pp. 103-117, 2020.

[4] K. Parvin, et al., "Particle Swarm Optimization Based Fuzzy Logic MPPT Inverter Controller for Grid Connected Wind Turbine," International Journal of Renewable Energy Research, vol. 9, no. 1, pp. 164-174, 2019.

[5] N. Peraino, et al., "Feasibility of Powering All Vehicles with Electricity from Solar and Wind Energy," Journal of Energy and Natural Resources, vol. 8, no. 4, pp. 127-136, 2019.

[6] E. A López, C. Ángeles-Camacho, and F. Bañuelos-Ruedas, "Advanced Methodology for Feasibility Studies on Building-Mounted Wind Turbines Installation in Urban Environment: Applying CFD Analysis,” Energy, vol. 167, no. 15, pp. 181-188, 2019.

[7] T. F. Ishugah, et al., "Advances in Wind Energy Resource Exploitation in Urban Environment: A Review," Renewable and Sustainable Energy Reviews, vol. 37, pp. 613-626, 2014.

[8] I. Hashem, Mohamed H. Mohamed, and Aida A. Hafiz, "Aero-Acoustics Noise Assessment for Wind-Lens Turbine," Energy, vol. 118, pp. 345-368, 2017.

[9] D. M. Magnus, L. L. Pfitscher, and C. C. Scharlau, "A synergy analysis of synthetic inertia and speed controllers on variable speed wind turbines," IEEE PES Innovative Smart Grid Technologies Conference-Latin America, pp. 1-6, 2019.

[10] G.H. Riahy, and M. Abedi, "Short Term Wind Speed Forecasting for Wind Turbine Applicationsusing Linear Prediction Method," Renewable Energy, vol. 33, no. 1, pp. 35-41, 2008.

[11] Y. Jiang, et al., "Very Short-Term Wind Speed Forecasting with Bayesian Structural Break Model," Renewable Energy, vol. 50, pp. 637-647, 2013.

[12] Y. Hong, Z. Song, and A. Kusiak, "Hour-Ahead Wind Power and Speed Forecasting Using Simultaneous Perturbation Stochastic Approximation (SPSA) Algorithm and Neural Network with Fuzzy Inputs," Energy, vol. 35, no. 9, pp. 3870-3876, 2010.

[13] R.G. Kavasseri, and K. Seetharaman, "Day-Ahead Wind Speed Forecasting Using F-ARIMA Models," Renewable Energy, vol. 34, no. 6, pp. 1388-1393, 2009.

[14] H. Liu, et al., "A Hybrid Statistical Method to Predict Wind Speed and Wind Power," Renewable Energy, vol. 35, no. 8, pp. 1857-186, 2010.

[15] W. K. Wong, M. Xia, and W. C. Chu, "Adaptive Neural Network Model for Time-Series Forecasting," European Journal of Operational Research, vol. 207, no. 2, pp. 807-816, 2010.

[16] E. Cadenas and W. Rivera, "Wind Speed Forecasting in Three Different Regions of Mexico, Using A Hybrid ARIMA-ANN Model,” Renewable Energy, vol. 35, no. 12, pp. 2732-2738, 2010.

[17] Kusiak, et al., "Anticipatory Control of Wind Turbines with Data-Driven Predictive Models," IEEE Transactions on Energy Conversion, vol. 24, pp. 766-774, 2009.

[18] I. Colak, et al., "Multi-time series and time scale modeling for wind speed and wind power forecasting part i: statistical methods, very short-term and short-term applications," Int. Conf. on Renewable Energy Research and Appl., 2015. 
[19] J. Zhang, and B. M. Hodge, "Forecastability as A Design Criterion in Wind Resource Assessment," Proceedings of the 8th International Conference on Foundations of Computer-Aided Process Design - FOCAPD, 2014

[20] E. Yatiyana, S. Rajakaruna, and A. Ghosh, "Wind Speed and Direction Forecasting for Wind Power Generation Using ARIMA Model," Australasian Universities Power Engineering Conference, 2017.

[21] G. W. Chang, et al., "A Hybrid Model for Forecasting Wind Speed and Wind Power Generation," IEEE Power and Energy Society General Meeting, 2016.

[22] S. S. Sanz, et al., "Short-Term Wind Speed Prediction by Hybridizing Global and Mesoscale Forecasting Models with Artificial Neural Networks," Eighth International Conference on Hybrid Intelligent Systems, pp. 608-612, 2008.

[23] D. Reddy, and S. Ramasamy, "A back-propagation network based MPPT algorithm for grid-tied wind energy system with vienna rectifier," International Journal of Renewable Energy Research, vol. 9, no. 2, pp.1097-1107, 2019.

[24] S. M. Lawan, et al., "Development of Wind Mapping Based on Artificial Neural Network (ANN) for Energy Exploration in Sarawak," International Journal of Renewable Energy Research, vol. 4, no. 3, pp.618-627, 201.

[25] S. A. Kalogirou, "Artificial Neural Networks in Renewable Energy Systems Applications: A Review," Renewable and Sustainable Energy Reviews, vol. 5, no. 4, pp. 373-401, 2001.

[26] W. Qiao and D. Lu, "A Survey on Wind Turbine Condition Monitoring and Fault Diagnosis-Part II: Signals and Signal Processing Methods," IEEE Transactions on Industrial Electronics, vol. 62, no. 10, pp. 6546-6557, 2015.

[27] K. F. Abdulraheem and G. Al-Kindi, "Wind Turbine Blade Fault Detection Using Wavelet Power Spectrum and Experimental Modal Analysis," International Journal of Renewable Energy Research, vol. 8, no. 4, pp.2167-2179, 2018.

[28] K. Wang, et al., "Deep belief network-based k-means cluster approach for short-term wind power forecasting," Energy, vol. 165, pp. 840-852, 2018.

[29] A. T. Sergio and T. B. Ludermir, "Deep Learning for Wind Speed Forecasting in Northeastern Region of Brazil," Brazilian Conference on Intelligent Systems (BRACIS), pp. 322-327, 2015.

[30] R. Y. Kazakova, et al., "Estimation of Wind Turbine Generator Model Parameters Using Artificial Intelligence Methods," Balkan Journal of Electrical and Computer Engineering, vol. 4, no. 2, pp. 51-57, 2016.

[31] M. N. Jyothi and P. R. Rao, "Very-Short Term Wind Power Forecasting Through Wavelet Based ANFIS," International Journal of Power Electronics and Drive Systems (IJPEDS), vol. 9, no. 1, pp. 397-405, 2018.

[32] M. M. Tumari, et al., "A modified grey wolf optimizer for improving wind plant energy production," Indonesian Journal of Electrical Engineering and Computer Science (IJEECS), vol. 18, no. 3, pp. 1123-1129, 2020.

[33] S. Barhmi and O. El Fatni, "Hourly Wind Speed Forecasting Based on Support Vector Machine and Artificial Neural Networks," International Journal of Artificial Intelligence, vol. 8, no. 3, pp. 286-291, 2019.

[34] M. Khodayar, O. Kaynak, and M. E. Khodayar., "Rough Deep Neural Architecture for Short-Term Wind Speed Forecasting," IEEE Transactions on Industrial Informatics, vol. 13, no. 6, pp. 2270-2779, 2017.

[35] X. Luo, et al., "Short-Term Wind Speed Forecasting via Stacked Extreme Learning Machine with Generalized Correntropy," IEEE Transactions on Industrial Informatics, vol. 14, no. 11, pp. 4963-4971, 2018.

[36] J. Fu, et al., "Condition Monitoring of Wind Turbine Gearbox Bearing Based on Deep Learning Model," IEEE Access, vol. 7, pp. 57078-57087, 2019.

[37] B. Dhaval and A. Deshpande, "Short-Term Load Forecasting with Using Multiple Linear Regression," International Journal of Electrical and Computer Engineering, vol. 10, no. 4, pp. 3911-3917, 2020.

[38] "NOAA's National Operational Model Archive and Distribution System (NOMADS)," NOAA, [Online]. Available: https://www.ncdc.noaa.gov/data-access/model-data/model-datasets.

\section{BIOGRAPHIES OF AUTHORS}

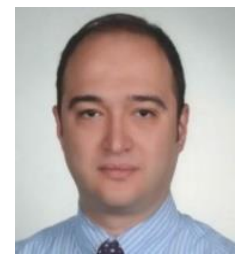

Gokhan Erdemir received his B.Sc., M.Sc. and Ph.D. degrees from Marmara University, Turkey, respectively. During his Ph.D., we worked as a research scholar at Michigan State University, Department of Electrical and Computer in East Lansing MI, USA. Now, he is an assistant professor at Istanbul Sabahattin Zaim University, Department of Electrical and Electronics Engineering. His research topics include robotics, control systems, and intelligent algorithms.

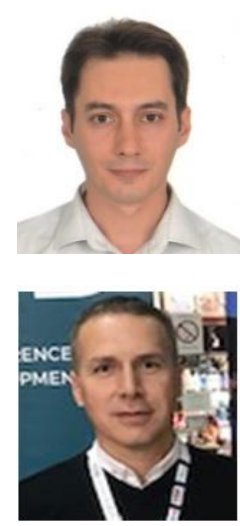

Aydın Tarık Zengin received his Electrical and Electronics Engineering B.S. degree from Ege University, Turkey, in 2007. Later on, received M.E. and Ph.D. degrees from Department of Computer Science and Electrical Engineering, Kumamoto University, Japan, in 2010 and 2013, respectively. Currently, he is an Asst. Prof. at Istanbul S. Zaim University. His research interests include autonomous systems and control theory.

T. Cetin Akinci received B.S degrees in Electrical Engineering. M.Sc. and Ph.D. degrees from Marmara University, Istanbul-Turkey. His research interests include artificial neural networks, deep learning, machine learning, image processing, signal processing and, data analysis. He has been working as an Associate Professor in Electrical Engineering Department of Istanbul Technical University (ITU) in Istanbul, Turkey. 